\title{
Investigation of formation mechanisms of chips in orthogonal cutting process
}

\author{
W. Ma \\ Institute of Mechanics, Chinese Academy of Sciences, P.R. China
}

\begin{abstract}
This work investigates the formation mechanisms of chips in orthogonal cutting of mild steel and the transformation conditions between various morphology chips. It is supposed that the modeling material follows the Johnson-Cook constitutive model. In orthogonal cutting process, both the plastic flow and the instability behaviors of chip materials are caused by the plane strain loadings. Therefore, the general instability behaviors of materials in plane strain state are first analyzed with linear perturbation method and a universal instability criterion is established. Based on the analytical results, the formation mechanisms of chips and the transformation conditions between continuous and serrated chips are further studied by instability phase diagram method. The results show that the chip formation strongly depends on the intensity ratios between shear and normal stresses. The ratios of dissipative rates of plastic work done by compression and shear stresses govern the transformation from continuous to serrated chips. These results are verified by the numerical simulations on the orthogonal cutting process.
\end{abstract}

\section{Introduction}

High speed cutting is a strong nonlinear and coupled thermomechanical process, in which the chip materials suffer from large plastic deformation, high strain rate, high temperature rise and severe friction. Nowadays, the cutting process has received much attention [1,2]; particularly, the instability behaviors of chip materials as adiabatic shear banding have been widely studied [3-10]. The formation of various morphology chips closely related with the cutting conditions [3-5]. For many metals, continuous chips are usually produced at a low cutting speed. As the cutting speed increases sufficiently, the chip shapes tend to be serrated [3]. Previous studies showed that whether continuous or serrated chip formation will depend on the plastic flow behaviors of chip materials [4-9]. Most studies on the cutting behaviors are, however, based on the single-shear plane model [4] and the card model [5]. In this aspect, the criteria for estimating the shear localization instability behaviors caused by simple shear loadings [10-12] are applied to describe the instability behaviors of chip materials. However, the studies on the transformation mechanisms between continuous and serrated chips are still lacking $[13,14]$. The recent study [15] based on the materials instability theory has shown that not only the shear stresses but also the compressive stresses in the region ahead of the tool tip strongly affect the mechanisms of chip formation and transformation.

For a better understanding of the mechanisms of chip formation and transformation in high speed cutting process, it is necessary to develop new analytical models to investigate the instability behaviors of materials. In recent years, the instability behaviors of materials under combined stress loading conditions have been studied and the instability criteria have been developed $[16,17]$. The transformation between continuous and serrated chips is found to be related to the dissipative rate of plastic work between compressive and shear deformation of chips [15]. These studies lay the groundwork for understanding the mechanisms of chip formation and transformation in cutting process, which is the focus of the present work.

In this paper, the material instability behaviors under plane strain state are first analyzed. Then the model of orthogonal cutting is established and the orthogonal cutting process is studied by numerical simulation. Through the comparison of numerical results with analytical results, we give the transformation condition between continuous and serrated chips in dissipative energy conception. Finally, the results are discussed and conclusions are summarized.

\section{Theory}

Consider a general plane strain body in the $x$ - $y$ coordinate plane as shown in Fig. 1. The impact loadings in the $x-y$ plane are assumed not to be changed in $z$ axial direction. All components of displacements and velocities of material particles as well as the components of stresses and strains are functions of spatial coordinates $x, y$ and time $t$. Our attention will focus on the instability of the plane body under the plane strain loading condition. The fundamental equations governing the material deformation consist of the equations of momentum and energy balance as well as the compatibility conditions.

For convenience, to rewrite these equations as

$$
\left\{\begin{array}{l}
\frac{\partial^{2} \varepsilon_{x}}{\partial y^{2}}+\frac{\partial^{2} \varepsilon_{y}}{\partial x^{2}}-\frac{\partial^{2} \gamma_{x y}}{\partial x \partial y}=0 \\
\rho \frac{\partial^{2} \gamma_{x y}}{\partial t^{2}}=\frac{\partial^{2}\left(\sigma_{x}+\sigma_{y}\right)}{\partial x \partial y}+\left(\frac{\partial^{2}}{\partial x^{2}}+\frac{\partial^{2}}{\partial y^{2}}\right) \tau_{x y} \\
\rho c \frac{\partial \theta}{\partial t}=\lambda\left(\frac{\partial^{2} \theta}{\partial x^{2}}+\frac{\partial^{2} \theta}{\partial y^{2}}\right)+K\left(\tau_{x y} \dot{\gamma}_{x y}+\sigma_{x} \dot{\varepsilon}_{x}+\sigma_{y} \dot{\varepsilon}_{y}\right)
\end{array}\right.
$$

where $\rho$ is mass density, $\mathrm{c}$ specific heat, $\lambda$ thermal conductivity, $\theta$ temperature and $\mathrm{K}$ the Taylor-Quinney coefficient. Suppose that the plastic flow of materials comply 


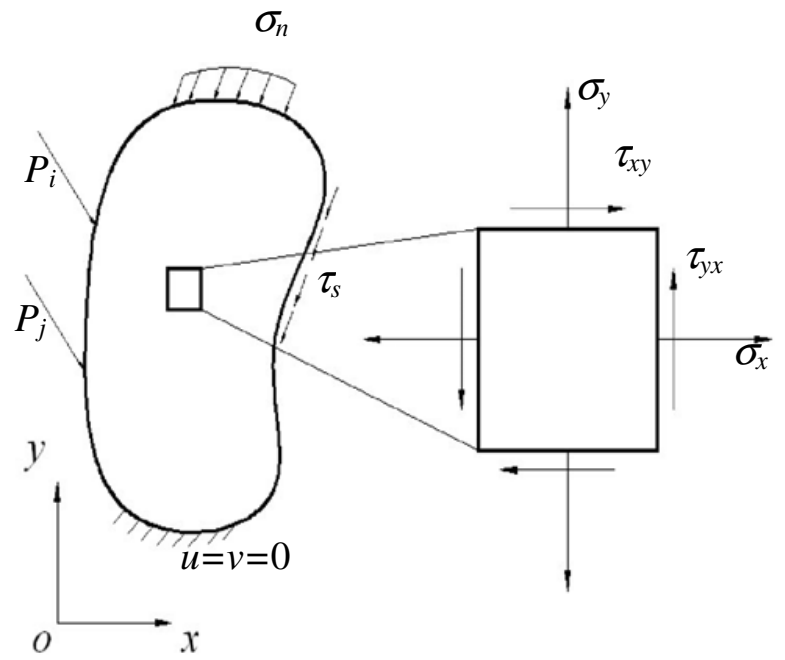

Fig. 1. The plane configuration with its loading conditions and stress state used in the present study.

with Johnson-Cook (J-C) constitutive model [18] which is given by

$$
\sigma=\left(A_{0}+B_{0} \varepsilon^{n}\right)\left(1+C_{0} \ln \frac{\dot{\varepsilon}}{\dot{\varepsilon}_{0}}\right)\left[1-\left(\frac{T-T_{r}}{T_{m}-T_{r}}\right)^{m}\right]
$$

where $\sigma$ and $\varepsilon$ are the Mises effective stress and plastic strain, respectively. $\dot{\varepsilon}$ effective plastic strain rate, $\dot{\varepsilon}_{0}$ reference strain rate, $T_{r}$ and $T_{m}$ reference temperature and melting point temperature. $A_{0}$ initial yield stress, $B_{0}$ work hardening stress, $n, C_{0}$ and $m$ coefficients of work hardening, strain-rate sensitivity and thermal softening. From relation (2), we can obtain the work hardening, strain-rate sensitivity and thermal softening by differential

$$
Q_{0}=\frac{\partial \sigma}{\partial \varepsilon}, \quad R_{0}=\frac{\partial \sigma}{\partial \dot{\varepsilon}}, \quad P_{0}=-\frac{\partial \sigma}{\partial T}
$$

In this study, the J-C model (2) to describe the plastic flow of workpiece material and the result of (3) is used to study the instability behaviors of chip materials caused by combined stresses.

The linear perturbation analysis is carried out to Eqs. (1) and (2). A tiny perturbation of the system is introduced by

$$
\Gamma_{i j}=\Gamma_{i j 0}+\delta \Gamma_{i j} e^{\alpha_{0} t+i k_{0}(x+y)} ; \quad \delta \Gamma_{i j} \ll \Gamma_{i j 0}
$$

where $\Gamma=\sigma, \tau, \varepsilon, \gamma, \theta . \Gamma_{i j 0}$ is a homogeneous solution of Eq. (1) and $\delta \Gamma_{i j}$ stands for the amplitude of perturbation, $k_{0}$ wave number and $\alpha_{0}$ perturbation growth rate in time of stresses, strain rates and temperature. Substituting Eq. (4) into the Eqs. (1) and (2), the spectral equation in dimensionless form is obtained as

$$
\begin{aligned}
& \alpha^{3}+\left[C+(A+1) k^{2}\right] \alpha^{2}+\left(A k^{2}+1-B\right) k^{2} \alpha+k^{4}+ \\
& \beta\left\{\left[(2 A+1) k^{2}+E\right] \alpha^{2}+\left[5 A k^{2}+2(1-B)+\frac{3}{2}(F-D)\right]\right. \\
& \left.k^{2} \alpha+\frac{3}{2} E k^{2}+5 k^{4}\right\}=0
\end{aligned}
$$

where $\beta$ is an index. When $\beta=0$, Eq. (5) is reduced into the spectral equation of simple shear case; when $\beta=1$, it is that of plane strain state case. In (5), the dimensionless variables below are introduced

$$
\begin{aligned}
& \alpha=\frac{\lambda \alpha_{0}}{c Q_{0}}, \quad k^{2}=\frac{\lambda^{2} k_{0}^{2}}{\rho c^{2} Q_{0}}, \quad A=\frac{c R_{0}}{\lambda}, \quad B=\frac{K P_{0} \tau_{x y 0}}{\rho c Q_{0}}, \\
& C=\frac{\lambda K P_{0} \dot{\gamma}_{x y 0}}{\rho c^{2} Q_{0}}, \quad D=\frac{K P_{0} \sigma_{k k 0}}{\rho c Q_{0}}, \quad E=\frac{\lambda K P_{0} \dot{\varepsilon}_{k k 0}}{\rho c^{2} Q_{0}}, \\
& F=\frac{K P_{0} R_{0} \dot{\varepsilon}_{k k 0}}{\rho c Q_{0}}, \quad \eta_{x}=\frac{\varepsilon_{x 0}}{\varepsilon_{k k 0}}, \quad \eta_{y}=\frac{\varepsilon_{y 0}}{\varepsilon_{k k 0}}
\end{aligned}
$$

where $\sigma_{k k 0}=\sigma_{x x 0}+\sigma_{y y 0}, \varepsilon_{k k 0}=\varepsilon_{x x 0}+\varepsilon_{y y 0}, \dot{\varepsilon}_{k k 0}=$ $\dot{\varepsilon}_{x x 0}+\dot{\varepsilon}_{y y 0}$.

The perturbation expression (4) shows that $\alpha>0$ is the required condition for material instability. For the long wavelengths $(k \rightarrow 0)$ or the short wavelengths $(k \rightarrow \infty)$, Eq. (5) just has the zero or negative roots, indicating that the plastic flow is constantly stable under these two extreme conditions.

Hence, instability is most likely to occur when $\alpha$ is positive and gets the maximum value $\alpha_{m}$. From the solution of Eq. (5) and the extreme condition $d \alpha_{m} / d k_{m}^{2}=$ 0 , we obtain two important functions as [15]

$$
\begin{aligned}
G_{1}= & 4\left(A \alpha_{m}+1\right)\left(\alpha_{m}+C\right)+4 \beta\left(A \alpha_{m}+1\right) \\
& \times\left[E+5\left(\alpha_{m}+C\right)+5 \beta E\right] \\
G_{2}= & \left\{\left[(A+1) \alpha_{m}-(B-1)\right]+\beta\left[(2 A+1) \alpha_{m}\right.\right. \\
& \left.\left.-2\left(B-1+\frac{3}{4} D-\frac{3}{4} F\right)+\frac{3}{2} E t_{c}\right]\right\}^{2}
\end{aligned}
$$

Similarly, in (7), when $\beta=0$, the functions $G_{1}$ and $G_{2}$ reduce to those corresponding to the simple shear state; while $\beta=1$, the functions $G_{1}$ and $G_{2}$ correspond to the plane strain state. From the two functions, the critical condition of material instability under the plane strain loading conditions is found as

$$
\xi P_{0}\left(2 \tau_{x y 0}+\sigma_{k k 0}-\frac{\lambda}{c} t_{c} \dot{\varepsilon}_{k k 0}-R \dot{\varepsilon}_{k k 0}\right)>2 \rho c Q_{0}
$$

The criterion (8) shows that the instability takes place when the thermal softening effect surpasses the work hardening effect. The normal stresses always enhance the thermal softening effect, and thus the plastic flow tends to instable; however, the normal strain rate, heat conduction and strain rate sensitivity reduce constantly the thermal softening effect and lead the plastic flow tend to stable.

Figure 2 is the instability phase diagram of materials under compression-shear combined stress loading conditions. In this figure, the phase points $Q_{i}$ demonstrate the instability conditions. The phase point $P$ in the area $O A B C$ represents the condition of shear localization instability caused by the simple shear loading. Hence, the phase points $Q_{i}$ in the area $O A B C$ denote the shear localization instability to being possible, and the $Q_{i}$ in the area $A D F B$ denote the conditions of nonlocalization thermal softening instability as necking.

If phase points $Q_{i}$ lay in the area with $\alpha<0$, the plastic flow is stable. Assuming that the stresses and strain rates in plane strain state proportionally change in magnitude, then the ratio of dissipative rates of plastic work done by shear 


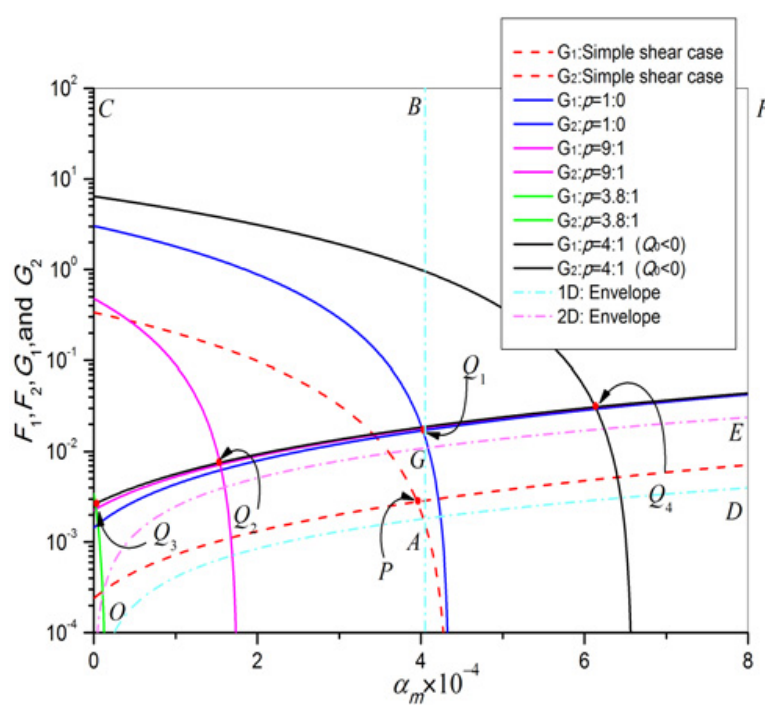

Fig. 2. The instability phase diagram of plastic flow of materials under plane loading conditions.

and normal stresses is given as

$$
p=\left|\tau_{x y 0} \dot{\gamma}_{x y 0}\right| /\left(\left|\sigma_{x 0} \dot{\varepsilon}_{x 0}\right|+\left|\sigma_{y 0} \dot{\varepsilon}_{y 0}\right|\right)
$$

In Fig. 2, the phase point $Q_{1}$ with $p=1: 0$ denotes the upper boundary condition of shear localization instability in the case of inhomogeneous simple shear loading. When $p$ equals to $9: 1$, the phase point $Q_{i}$ shifts towards the left to $Q_{2}$ in area $O A B C$, meaning the shear localization instability is possible; note that the value of growth rate $\alpha$ decreases from 4.02 to 1.54 , so that the adiabatic shear instability becomes more difficult. When the ratio $p=$ 3.8:1, $Q_{i}$ moves to $Q_{3}$ on the $y$ axis. The $Q_{3}$ denotes the transformation condition among various plastic deformation modes. If the ratio $p$ increases continuously and material remains in strain hardening stage $\left(Q_{0}>0\right)$, the phase point $Q_{i}$ will move into the area with $\alpha<$ 0 , implying plastic flow is stable. If the strain softening occurs $\left(Q_{0}<0\right), Q_{i}$ will move reversely towards the right and arrive to $Q_{4}$. The instability condition denoted by $Q_{4}$ describes nonlocalization thermal softening instability mainly caused by compressive strain softening. Therefore, under the compression-shear loading conditions, the ratio $p=3.8: 1$, as a criterion, can be used to estimate the plastic flow and instability behaviors of materials.

\section{Simulation of orthogonal cutting process and discussion}

The plot in Fig. 3 models the orthogonal cutting process. Tool moves relative to workpiece at the speed of $V_{c} . L, H$, $h$ are workpiece length, width and cutting depth. The tool material is treated as rigid body. The friction between tool and chip follows Coulomb friction law. The flow behavior of workpiece material satisfies J-C model. The force and

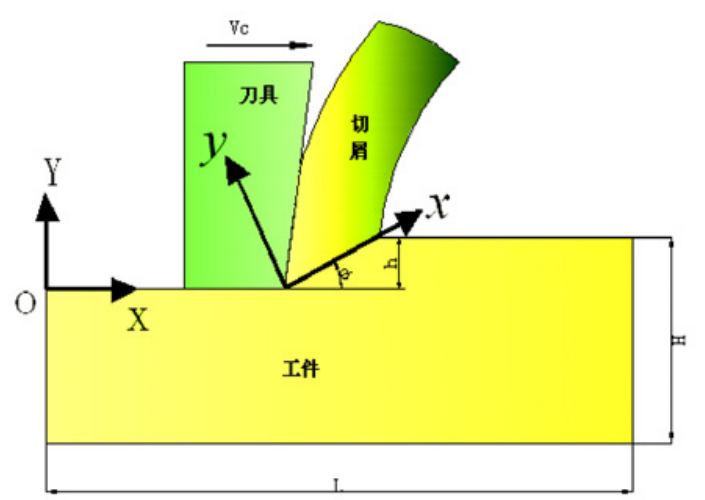

Fig. 3. Schematic of orthogonal cutting model of materials.

displacement boundary conditions are

$$
\begin{cases}\sigma_{n}=\sigma_{T}, \tau_{s}=\mu \sigma_{n}, & X=\mathrm{V}_{\mathrm{c}} t+l \sin \alpha_{R}, Y=l \cos \alpha_{R} \\ u_{X}=0, & X=0, h-H<Y<0 ; X=L, \\ & h-H<Y<h \\ \sigma_{Y}=0, \tau_{X Y}=0, & 0<X<L, Y=h \\ u_{X}=0, u_{Y}=0, & 0<X<L, Y=h-H\end{cases}
$$

where $l$ is the contact distance in tool-chip interface, $\alpha_{R}$ rake angle, $\sigma_{n}$ normal stress, $\tau_{s}$ shear stress, $\sigma_{T}$ tool normal stress. The temperature boundary conditions are

$$
\begin{cases}\frac{\partial T}{\partial X}=0, & X=0, h-H<Y<0 ; X=L, \\ \frac{\partial T}{\partial Y}=0, & 0<X<L, Y=h ; 0<X<L, \\ k_{h} \frac{\partial T}{\partial X}{ }^{\partial T_{T}}=0, & Y=V_{c} t, 0<Y<h: \\ +k_{T} \frac{}{\partial X}=0, & \end{cases}
$$

where $k_{h}$ is the thermal conductivity of the workpiece, $k_{T}$ thermal conductivity of tool, $T_{T}$ temperature of tool. Numerical simulation is performed by ABAQUS/Explicit. The cutting depth is $0.1 \mathrm{~mm}$. For considering the thermomechanical coupled effect, four node plane strain coupled temperature-displacement element CPE4RT is adopted. The total element numbers is 14000 .

The simulating results are shown in Fig. 4 and 5. We can see that, when the cutting speed is $25 \mathrm{~m} / \mathrm{s}$, the continuous chips are produced, and as the cutting speed increases to $50 \mathrm{~m} / \mathrm{s}$, the serrated chips fully develop. The results agree with the experimental observations of [19] in which the transformation speed of chip morphology for mild steel is about $38 \mathrm{~m} / \mathrm{s}$.

Figure 4 shows the contour plots of Mises effective stress, shear stress, shear strain and temperature when the cutting speed is $25 \mathrm{~m} / \mathrm{s}$. The Mises effective stress distributes in the extensive zone including the primary shear zone (PSZ) and secondary shear zone (SSZ) (Fig. 4(a)) The peak effective stress with intensity $1.38 \mathrm{GPa}$ appears in the chip free surface of PSZ and the top of SSZ. By comparing the stress contour plots in Fig. 4(a) and 4(b), we can see that the chip material in the SSZ mainly suffer from the compressive stress produced by tool. The shear stress 


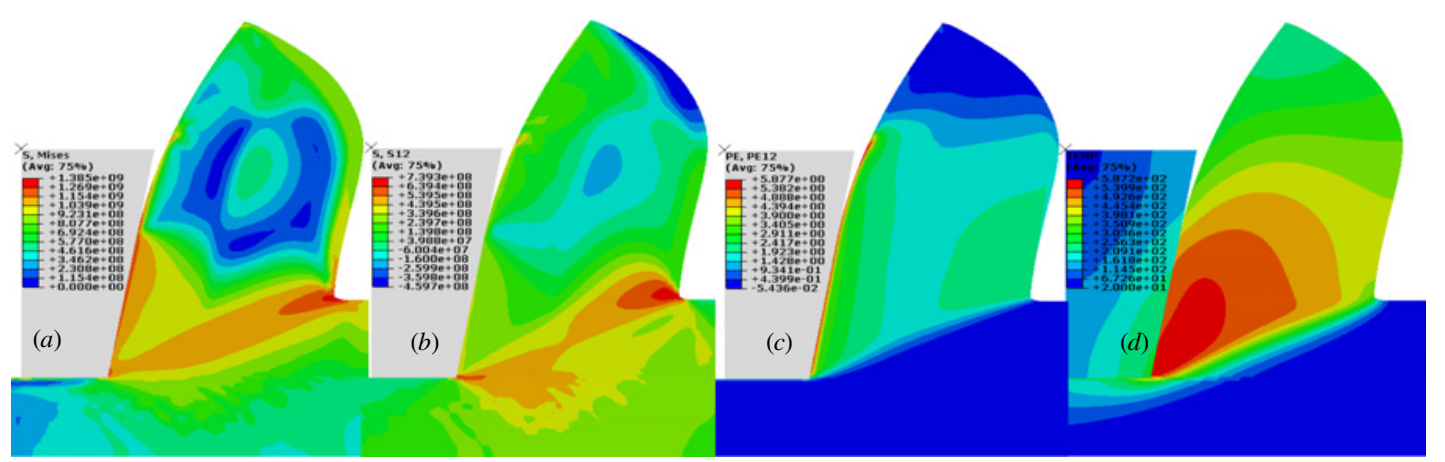

Fig. 4. The contours of Mises effective stress (a), shear stress (b), shear strain (c) and temperature (d) at the cutting speeds of $25 \mathrm{~m} / \mathrm{s}$.

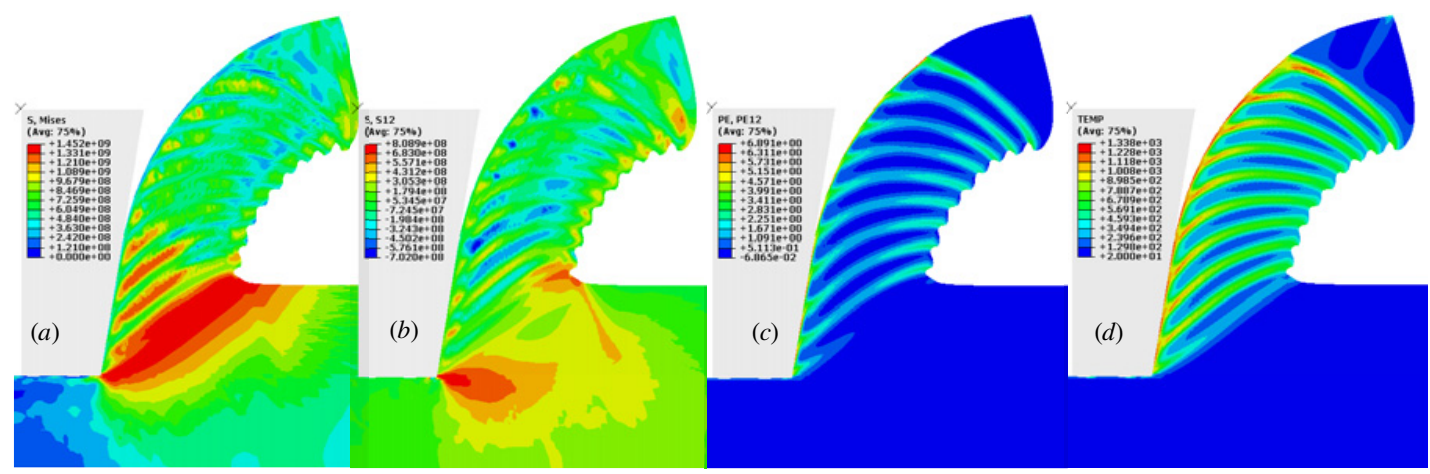

Fig. 5. The contours of Mises effective stress (a), shear stress (b), shear strain (c) and temperature (d) at the cutting speeds of $50 \mathrm{~m} / \mathrm{s}$.

with the peak intensity 7.4 MPa presents in a large region which embraces PSZ and extends into the uncut workpiece materials. In the region of uncut workpiece the shear stress intensity is $4.4 \mathrm{MPa}$. Thus, the distribution of shear stress tends to nonlocalization. The shear strain contour in Fig. 4(c) shows that the plastic deformation of chip materials is nearly uniform and the average shear strain is about 3 . The heat affecting zone of high temperature also includes the PSZ and SSZ and the peak temperature in chip materials is about $590 \mathrm{~K}$ (Fig. 4(d)). The high temperature rise is caused by the heat converted from plastic work done. The nonlocalization plastic deformation and thermal softening undergone by chip materials indicate that the formation process of continuous chip is a long range steady plastic flow process induced by tool compression-shear combined stresses. Consequently, in the machining of lower cutting speeds, the chip materials mainly suffer from the nonlocalization thermal softening and steady plastic flow, in which the steady evolution in stress, deformation and temperature takes place.

Figure 5 presents the simulating results as the cutting speed increases to $50 \mathrm{~m} / \mathrm{s}$. With increasing the cutting speed, chips change from continuous to serrate. Similarly, the Mises effective stress distributes in the extensive zone embracing the PSZ and SSZ and extends into the uncut chip (Fig. 5(a)). Inside the PSZ, the peak intensity of effective stress is $1.45 \mathrm{GPa}$; whereas the shear stress intensity is less than $0.81 \mathrm{GPa}$ (Fig. 5(b)), suggesting that the compressive stresses in PSZ affect strongly the shear banding formation in chips. Note that these peak shear stress presents in the zone ahead of tool tip (Fig. 5(b)); hence, it is the compressive-shear combined stresses at the tool tip that cause the separation of chip material from

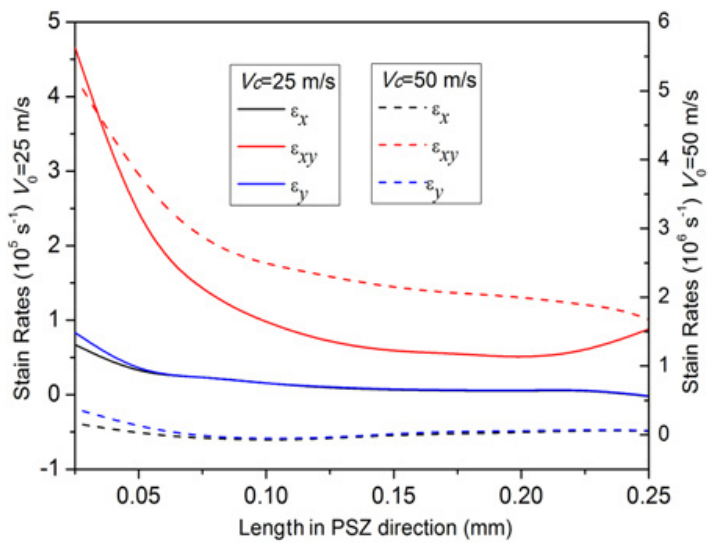

Fig. 6. Strain rate distributions in the primary shear direction with different cutting speeds.

the uncut workpiece. The chip materials undergo evident shear localization deformation and the peak shear strain in the localized deformation zone arrives 3.5 (Fig. 5(c)). The high temperature rise is also limited in the localized deformation zone and the peak temperature is near $900^{\circ} \mathrm{C}$ (Fig. 5(d)). Therefore, in the high speed cutting process, the localization thermal softening takes place in chip materials and the adiabatic shear localization instability cause the serrated chip formation.

Figure 6 demonstrates the variations of strain rates along the primary shear direction at the cutting speeds of 25 and $50 \mathrm{~m} / \mathrm{s}$. The values of shear strain rate at high cutting speed are one order larger than those in low cutting speed. In the PSZ, the normal strain rate is much smaller than the shear strain rate; thus, the shear deformation is 


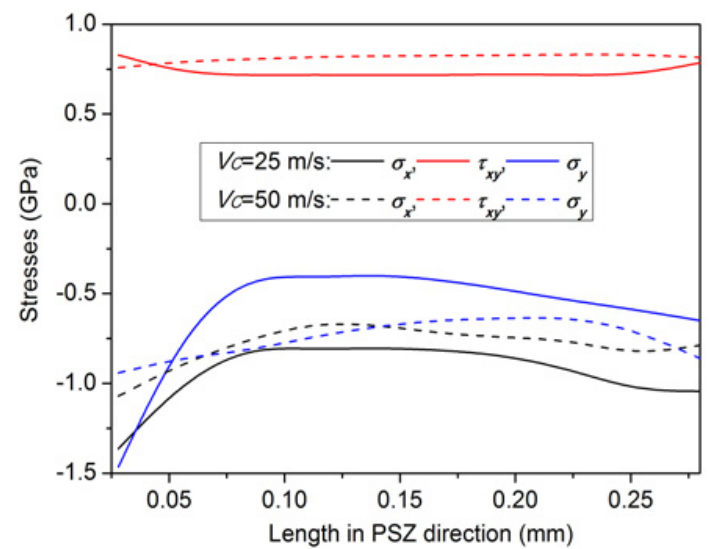

Fig. 7. Stress distributions in the primary shear direction with different cutting speeds.

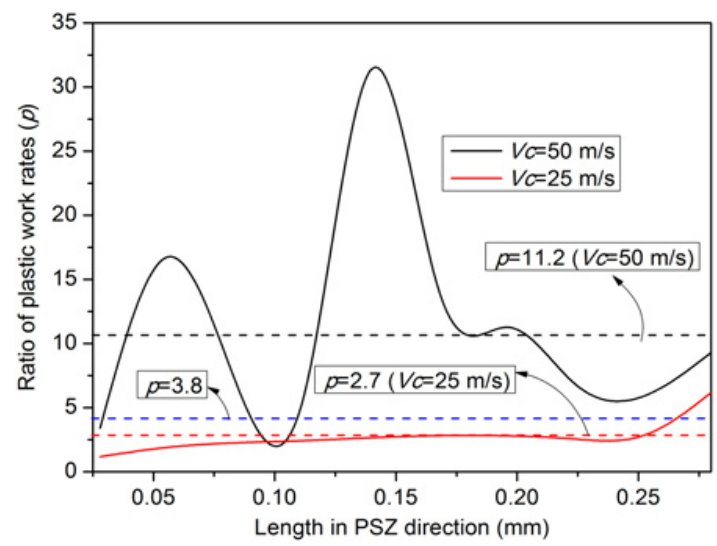

Fig. 8. Distribution of dissipative rates plastic work in the primary shear direction with different cutting speeds.

dominative mehcanism of plastic flow. Figure 7 shows the distributions of stresses in the primary shear direction. The compressive stresses uniformly distribute in the PSZ and the intensities approach to $0.8 \mathrm{GPa}$. From the modeling results of stress and strain rates, the ratios of dissipative rates of plastic work $p$ can be determined as shown in Fig. 8. It can be noticed from the figure that, the ratios vary sharply with the positions in the primary shear direction as the cutting speed is $50 \mathrm{~m} / \mathrm{s}$. However, the average value is found to be equals to 11.2 which is larger than the theoretical prediction value 3.8; that is, the plastic deformation of chip satisfies the critical transition condition of stable plastic flow and various instability modes of materials. Thus, the shear localization instability occurs in chip materials and the chip becomes serrated. At the cutting speed of $2.5 \mathrm{~m} / \mathrm{s}$, the average value of $p$ is 2.7 that is less than the critical value 3.8 , implying that the chip materials undergo homogeneous plastic flow and no shear localization instability occurs, and thus the chip is continuous. We have seen that, in the orthogonal cutting process, the compressive stresses on the primary shear plane strongly affect the transformation mechanisms of chip morphology.

\section{Conclusion}

A general criterion for estimating material instability under plane strain loading condition has been established, which describes the instability behaviors of materials with strain hardening, strain rate sensitivity and thermal softening, and considers the effects of heat conduction, normal stresses and strain rates and loading conditions on instability mechanisms. This criterion indicates that, when the thermal softening effect of material plastic deformation surpasses the work hardening, the material instabilities take place and the modes of material instability are likely to be the shear localization and the non-localized thermal softening.

The simulating results on the orthogonal cutting process of mild steel show that the chip morphology depends on the cutting speeds strongly. At lower cutting speed, the nonlocalization heat effect on plastic flow behavior is evident. The chip material undergoes nonlocalization thermal softening and long range homogeneous plastic flow which results in the formation of continuous chip. With increasing the cutting speed, localization thermal softening controls the plastic flow and the chip material undergoes adiabatic shear localization instability. Serrated chip develops.

\section{Acknowledgements}

The authors acknowledge financial supports from the National Nature Science Foundation of China (Grant No. 10972227 and 11132011) and the National Key Basic Research Program of China (Grant No. 2009CB724401).

\section{References}

1. M.C. Shaw, 1984. Metal Cutting Principles, Clarendon Press, Oxford.

2. P. L. B. Oxley, 1989. The Mechanics of Machining: An Analytical Approach to Assessing Machinability. Ellis Horwood Limited Publishers, Chichester.

3. R. Komanduri, T. Schroeder, J. Hazra, B. F. von Turkovich, D. G. Flom. On the catastrophic shear instability in high-speed machining of an AISI 4340 steel. Journal of Engineering for Industry, 1982, 104: $121 \sim 131$

4. M. Eugene Merchant. Mechanics of the metal cutting process. I. orthogonal cutting and a type 2 chip. Journal of Applied Mechanics, 1945, 16: 267 275.

5. Piispanen, V. Theory of formation of metal chips. Journal of Applied Physics, 1948, 19: 876 881.

6. D. Buryta, R. Sowerby, I. Yellowley. Stress distribution on the rake face during orthogonal machining. Int. J. Mach. Tools Manufact, 1994, 34: 721 739.

7. M. A. Davies, A. L. Cooke and E. R. Larsen. High bandwidth thermal microscopy of machining AISI 1045 steel. CIRP, Annals-Manufacturing Technology. 2005, 54(1): 63 66

8. T. D. Marusich, M. Ortiz. Modelling and simulation of high-speed machining. Int. J. Num. Meth. Eng, 1995, 38: $3675 \sim 3694$ 
9. A. J. Shih. Finite element simulation of orthogonal cutting. Journal of Engineering for Industry, 1995, 117: 84 93.

10. R.F. Recht. Catastrophic thermoplastic shear. Journal of Applied Mechanics, 1964, 31: 189 193.

11. R. J.Clifton, 1980. Report to the NRC Committee on Material Responses to Ultrasonic Loading Rates.

12. Y.L. Bai, Thermo-plastic instability in simple shear. J. Mech. Phys. Solids C 1982, 30: 195 207

13. T.J. Burns, and M.A. Davies. 1997, Nonlinear Dynamics Model for Chip Segmentation in Machining. 79, 447.

14. Molinari, A., Musquar, C., Sutter, G., 2002. Adiabatic shear banding in high speed machining of Ti-6Al-4V: experiments and modeling. Int. J. Plast. 18, 443.

15. W. Ma, X. W. Li, L. H. Dai, L. Ling, 2012. Instability criterion of materials in combined stress states and its application to orthogonal cutting process. Int. J. Plast., 30-31, 18 .

16. C. Fressengeas, A. Molinari. Instability and localization of plastic flow in shear at high strain rates. J. Mech. Phys. Solids, 1987, 35(2): 185 211.

17. L. Anand, K. H. Kim, T. G. Shawki. Onset of shear localization in viscoplastic solids. J. Mech. Phys. Solids, 1987, 35(4): 407 429.

18. Gordon R. Johnson, William H. Cook. 1985. Fracture characteristic of three metals subjected to various strains, strain rates, temperatures and pressures. Engineering Fracture Mechanics, 21(1): 31 48.

19. G. G. Ye, S. F. Xue, W. Ma, M. Q. Jiang, Z. Ling, X. H. Tong, L. H. Dai. 2012. Cutting AISI 1045 steel at very high speeds. International Journal of Machine Tools \& Manufacture. 56, 1-9. 